\title{
Manejo perioperatorio del paciente con COVID-19 con afección cardíaca, renal y neurológica
}

\author{
Perioperative management of the COVID-19 patient \\ with cardiac, renal and neurological conditions
}

\author{
Dr. José Manuel Portela-Ortiz,* Dra. Gabriela Garza-Benavides, \\ Dra. Delia Brenda Paola Ocampo-Valenciall
}

\begin{abstract}
RESUMEN. En diciembre de 2019, se produjo un brote de la enfermedad por coronavirus 2019 (COVID-19) en Wuhan, China, y se extendió rápidamente a otras áreas en todo el mundo. Aunque el daño alveolar difuso y la insuficiencia respiratoria aguda fueron las características principales, es necesario explorar la participación de otros órganos. Realizamos esta revisión bibliográfica para evaluar la última evidencia sobre la asociación entre la enfermedad cerebrovascular, la enfermedad cardiovascular y renal, y el mal pronóstico en pacientes con neumonía por enfermedad por coronavirus 2019 (COVID-19). La afección cardiovascular, cerebrovascular y renal se asociaron con una mayor mortalidad y un aumento en la gravedad del COVID-19. El género, la edad, la hipertensión, diabetes y comorbilidades respiratorias influyeron poco en la asociación.
\end{abstract}

ABSTRACT. In December 2019, a coronavirus 2019 (COVID-19) disease outbreak occurred in Wuhan, China, and rapidly spread to other areas worldwide. Although diffuse alveolar damage and acute respiratory failure were the main features, the involvement of other organs needs to be explored. We conducted the review to evaluate the latest evidence on the association between cerebrovascular, cardiovascular and kidney disease, and poor outcome in patients with coronavirus disease 2019 (COVID-19) pneumonia. Cardiovascular, cerebrovascular and kidney disease was associated with increased mortality and borderline increase in severity of COVID-19. Gender, age, hypertension, diabetes, and respiratory comorbidities did not influence the association.

\section{INTRODUCCIÓN}

D esde su inicio en diciembre de 2019 en China el coronavirus se ha expandido a nivel mundial. En febrero de este año, la Organización Mundial de Salud lo llamó «COVID-19» y al virus responsable de esta enfermedad se le conoce como Severe acute respiratory syndrome coronavirus 2 (SARS-CoV-2) por la Comisión Internacional de Taxonomía Virus ${ }^{(1)}$.

Se han propuesto los siguientes criterios diagnósticos para COVID-19: fiebre y síntomas respiratorios ( $\mathrm{Fr}>30 \mathrm{rpm}$, $\mathrm{SatO}_{2} \leq 93 \%$ y $\mathrm{PaO}_{2} / \mathrm{FiO}_{2} \leq 300 \mathrm{mmHg}$ ), leucopenia, linfopenia, TAC con anormalidades radiológicas; y se necesitan $\geq 2$ de estos criterios además de un PCR positivo para confirmarlo ${ }^{(2,3)}$.

El SARS-CoV-2 se disemina por aerosoles y gotas. Se sabe que se mantiene hasta tres horas en su forma de aerosol, hasta 24 horas en superficies sólidas y puede durar tres días en plásticos o metal. El tiempo medio de incubación es de cuatro a cinco días y se pueden presentar síntomas hasta 11.5 días posterior a la exposición ${ }^{(3)}$.

En este artículo daremos recomendaciones basadas en pacientes que tienen alteraciones principalmente a nivel cardíaco, renal y neurológico.

Cuando el paciente COVID-19, o con sospecha, llega con estas afecciones no está de más saber que necesitamos todo el equipo de protección necesario que implica bata impermeable, gorro, lentes de protección impermeables, guantes, cubrebocas N95, y el entrenamiento de
Anestesiología

Octubre-Diciembre 2020

Vol. 43. No. 4. pp 241-244

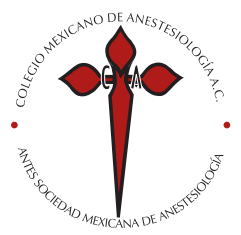

Palabras clave: COVID-19, pandemia, enfermedad cardiovascular, accidente cerebrovascular, enfermedad renal, neumonía, mortalidad.

Keywords:

COVID-19, pandemic, cardiovascular disease, cerebrovascular disease, renal disease, pneumonia, mortality.

\footnotetext{
* Médico adscrito. Hospital Ángeles Pedregal.

‡ Jefe del Servicio de Anestesiología.

$\S$ Residente de tercer año de Anestesiología. Hospital

Ángeles Pedregal

\| Facultad Mexicana de Medicina.

Universidad La Salle.
}

Solicitud de sobretiros: Dra. Gabriela Garza-Benavides E-mail: gabbygarza.92@hotmail.com

Recibido para publicación: 08-06-2020

Aceptado para publicación: 06-07-2020 
cómo se deben colocar y retirar cada uno de estos elementos. Además de realizar el lavado de manos cada que sea necesario, evitar contacto con ojos, nariz y boca, mantener la distancia cuando sea posible, estornudar o toser con codo en flexión, entre los más importantes ${ }^{(1)}$.

Las recomendaciones proporcionadas en este documento reflejan la opinión de consenso de expertos basada en la información disponible en el momento de la redacción y están sujetas a cambios a medida que aumente el conocimiento ${ }^{(4)}$.

\section{AFECCIÓN RENAL}

La mayoría de los pacientes infectados por COVID-19 tienen comorbilidades como hipertensión arterial, diabetes mellitus y enfermedades cardiovasculares ${ }^{(1)}$.

Como definición se conoce que la lesión renal aguda es el incremento de creatinina $0.3 \mathrm{mg} / \mathrm{dL}$ en las próximas 48 horas o aumento de $50 \%$ de creatinina comparada con la basal en los próximos siete días ${ }^{(2)}$.

El SARS-CoV-2 se une al receptor de la enzima convertidora de angiotensina 2 y a la dipeptil-peptidasa 4 (CD26) para su interiorización en la célula. Estos receptores se encuentran a nivel renal, generando mayor riesgo en los pacientes con lesión renal o crónica.

Por lo tanto, la gravedad y el riesgo de contraer COVID-19 depende de la unión a los receptores de ECA $2^{(1-3)}$.

Existen otros factores relacionados como el desequilibrio de células CD4+/CD8+, la actividad disminuida de células Natural Killer, alteración de pequeños vasos y capilares por SARS-CoV-2 por citosinas inflamatorias ${ }^{(1)}$.

La enfermedad de COVID-19 es más grave y se presenta de manera más agresiva en los adultos mayores que cursan con disminución de la función renal ${ }^{(1)}$, hay que evitar fármacos nefrotóxicos y tratar de dar un adecuado soporte hemodinámico. No se ha encontrado fundamento científico para retirar medicamentos antihipertensivos como los inhibidores de la enzima convertidora de angiotensina y los antagonistas de la angiotensina ${ }^{(2,5,6)}$.

En un metaanálisis Jun y colaboradores encontraron que el riesgo general de AKI en todos los pacientes hospitalizados parecía ser bajo (incidencia de 3\%). Este riesgo aumenta a $19 \%$ cuando los pacientes ingresan a UTI. También se observa que la necesidad de terapia de reemplazo renal se incrementa en $13 \%$ en los pacientes que requieren de cuidados intensivos. Tomando en cuenta que la mayoría de los estudios incluidos son de China, los resultados de este metaanálisis pueden no ser aplicables a otras regiones del mundo ${ }^{(7)}$.

Algunos pacientes con enfermedad renal crónica (ERC) necesitan hemodiálisis y los centros que llevan a cabo este procedimiento pueden ser vectores para esparcir la pandemia ${ }^{(1)}$.

Una serie de casos evaluó la función del injerto en admisión de pacientes con diagnóstico confirmado de COVID-19; en términos de estimación, la tasa de filtración glomerular fue de $39.9 \pm 24.5 \mathrm{~cm}^{3} / \mathrm{min}$. El mismo estudio evaluó las TAC de tórax sin contraste realizadas por protocolo. De acuerdo a éstas, el patrón más común de afección pulmonar fue bilateral, con un patrón difuso y una distribución segmentaria posterior. Los autores concluyen que las imágenes por TAC pueden tener un papel importante en la predicción de resultados por COVID-19 para pacientes receptores de transplante ${ }^{(8)}$.

Por último, las recomendaciones generales a pacientes con ERC: lavado de manos y evitar contacto con ojos, nariz y boca; distancia, estornudo o tos con codo en flexión; no tocar superficies de lugares públicos; seguir con medicación (IECAs, glucocorticoides e inmunosupresores), dieta (cereales, verduras y frutas, proteínas -0.6 a $0.8 \mathrm{~g} / \mathrm{kg} / \mathrm{día}$; sal, potasio, fósforo y purinas), manejo psicológico ${ }^{(1)}$.

\section{AFECCIÓN CARDÍACA}

La progresión de la enfermedad de manera aguda se divide en tres fases: la fase temprana, la fase pulmonar y la fase de hiperinflamación. La mayoría de los casos son asintomáticos (81\%). Durante la fase temprana de la infección, el virus se infiltra en el parénquima pulmonar y empieza a proliferar, esta etapa se caracteriza por síntomas constitucionales leves e inicia la fase de inmunidad dada por monocitos y macrófagos y caracterizada por: vasodilatación, permeabilidad endotelial y reclutamiento leucocitario, generando daño pulmonar, hipoxemia, estrés cardiovascular, cursando con linfocitopenia (linfocitos T helper y reguladores) y neutrofilia. La segunda fase o pulmonar donde se pueden ver las imágenes anormales y, por último, la fase de respuesta sistémica se caracteriza por la falla orgánica múltiple y elevación de biomarcadores inflamatorios ${ }^{(9)}$.

Entre los biomarcadores elevados se encuentra la troponina I y el péptido natriurético cerebral siendo marcadores de mal pronóstico. El daño al miocardio y la falla cardíaca conllevan a $40 \%$ de muertes con o sin afección pulmonar ${ }^{(9)}$. El daño es multifactorial mediado por el estrés cardíaco, la falla respiratoria e hipoxemia, daño directo al miocardio por el virus y lesión indirecta por la respuesta inflamatoria sistémica. Las autopsias reportan infiltrado de macrófagos y células CD4. Estos infiltrados monocíticos se asocian a necrosis cardiomiocítica satisfaciendo los criterios de Dallas de miocarditis. Las manifestaciones cardiovasculares en los pacientes con COVID-19 son complejas y pueden presentarse alteraciones en la presión arterial, arritmias, infarto agudo al miocardio (anormalidades electrocardiográficas, elevación de troponinas y dolor torácico), miocarditis simulando síndrome coronario agudo, cardiomiopatía por estrés, cardiomiopatía no isquémica, espasmo coronario, lesión miocárdica, entre otras ${ }^{(9,10)}$.

En la actualidad no existen fármacos aprobados que hayan demostrado eficacia en el tratamiento del COVID. Los estudios 
publicados a la fecha tienen serios defectos metodológicos, y carecen de una revisión adecuada por revisores calificados.

Por lo tanto, al intentar el uso compasivo de algunos fármacos deberán vigilarse especialmente los efectos colaterales de los mismos en pacientes con afección cardíaca( ${ }^{(3,9,11)}$.

Los pacientes con COVID-19 pueden mostrar un síndrome de dolor precordial y elevación del ST, esto puede deberse a la inestabilización de una placa ateromatosa o presentarse en pacientes con coronarias prácticamente normales.

Otras formas de presentación son las de cardiomiopatía por estrés, arritmias o bajo gasto cardíaco, por lo que deberemos tener en cuenta las variadas presentaciones clínicas producidas por este agente viral.

Es importante recalcar que de los pacientes con síndrome coronario agudo, sólo 50\% reperfunden con fibrinólisis requiriendo posteriormente la intervención coronaria percutánea. Se recomienda efectuar estos procedimientos con la protección necesaria ${ }^{(12)}$.

Cada vez que se realiza cateterismo cardíaco, hay que tener en cuenta las recomendaciones de protección general ya descritas en la introducción para pacientes con COVID-19.

\section{AFECCIÓN NEUROLÓGICA}

Los coronavirus son patógenos conocidos con potencial neuroinvasivo. Algunos pacientes pueden mostrar signos neurológicos y síntomas que van desde cefalea, náuseas, vómitos y confusión hasta anosmia, ageusia, encefalitis y accidente cerebrovascular ${ }^{(4)}$.

Los pacientes COVID-19 positivos tienen un riesgo aumentado de ataque cerebrovascular isquémico agudo. Un reporte reciente de China sugiere que los síntomas neurológicos como mareos, cefalea, hipogeusia e hiposmia son comunes (36\%) en pacientes con COVID-19.

Encefalopatía y alteraciones en el estado mental se han reportado en pacientes infectados con el virus SARS-CoV-2.

Una serie de casos realizada en Wuhan, China detectó que de 214 pacientes COVID-19 positivos, $36.4 \%$ tenía manifestaciones neurológicas en la presentación inicial, incluyendo cefalea, náusea, vómito, alteración de la conciencia, ataxia, enfermedad cerebrovascular aguda y convulsiones.

La enfermedad cerebrovascular es más común en COVID-19 grave. Un reporte de 221 pacientes provenientes de Wuhan, China detectó $5 \%$ de incidencia de evento cerebrovascular isquémico y $1 \%$ de incidencia de hemorragia cerebral.

Adicionalmente, la cardiotoxicidad directa e indirecta secundaria a la excesiva estimulación sistémica proinflamatoria (tormenta de citoquinas), hipercoagulabilidad, e invasión directa del miocardio están asociadas a infarto agudo al miocardio, insuficiencia cardíaca y arritmias, que son factores de riesgo importantes de accidente cerebrovascular.

La pandemia por COVID 2019 tiene implicaciones graves y relevantes para el neuroanestesiólogo, incluyendo las manifestaciones neurológicas de la enfermedad, el impacto de proveer anestesia para procedimientos neuroquirúrgicos específicos y terapia electroconvulsiva, y el bienestar del proveedor de salud ${ }^{(13)}$.

Por último, $38 \%$ de los pacientes con complicaciones cerebrovasculares fallecen. En conjunto, estos reportes preliminares sugieren que los pacientes con COVID-19 podrían presentarse con mayor frecuencia para el tratamiento endovascular del accidente cerebrovascular isquémico agudo y también podrían tener un riesgo elevado de accidente cerebrovascular perioperatorio si requieren una cirugía durante la infección aguda ${ }^{(14)}$.

En el caso de los procedimientos de radiología neurointervencionista que sean urgentes, en los que no es posible realizar la prueba, el paciente debe ser tratado como sospechoso de COVID-19 positivo ${ }^{(15)}$.

\section{CONCLUSIÓN}

Conocer las implicaciones del paciente COVID-19 es importante para nuestro manejo anestésico, además de valorar el bienestar de los proveedores de atención médica; la noción de autosacrificio es un gran error. El éxito de controlar y erradicar una pandemia depende de la disponibilidad de los recursos, incluido el personal. Médicos, enfermeras y demás personal de salud pueden no darse cuenta de que durante una pandemia la naturaleza del «paciente» cambia del individuo a una comunidad. Por lo tanto, reforzamos la importancia de la máxima seguridad para preservar los recursos humanos necesarios para superar la pandemia. El bienestar del personal de salud debe abarcar la salud física y mental ${ }^{(16)}$. 


\section{REFERENCIAS}

1. Li J, Li S, Zhao L, Kong D, Guo Z. Management recommendations for patients with chronic kidney disease during the novel coronavirus disease 2019 (COVID-19) epidemic. Chronic Dis Transl Med. 2020;6:119-113.

2. Cheng Y, Luo R, Wang K, Zhang M, Wang Z, Dong L, et al. Kidney disease is associated with in-hospital death of patients with COVID-19. Kidney Int. 2020;97:829-838.

3. Alemzadeh-Ansari M. Coronavirus disease 2019 (COVID-19) and cardiovascular events. Res Cardiovasc Med. 2020;9:1-2.

4. Pérez CA. Looking ahead: the risk of neurologic complications due to COVID-19. Neurol Clin Pract 2020.. 10.1212/CPJ.0000000000000836; doi: 10.1212/cpj.0000000000000836.

5. Saiz FL. Inhibidores del Sistema Renina-Angiotensina como potencial terapia frente al COVID-19. 2020;19:1-4.

6. Klimas J, Olvedy M, Ochodnicka-Mackovicova K, Kruzliak P, Cacanyiova S, et al. Perinatally administered losartan augments renal ACE2 expression but not cardiac or renal Mas receptor in spontaneously hypertensive rats. J Cell Mol Med. 2015;19:1965-1974.

7. Ng JJ, Luo Y, Phua K, Choong AM. Acute kidney injury in hospitalized patients with coronavirus disease 2019 (COVID-19): a meta-analysis. J Infect. 2020;10-13. doi: 10.1016/j.jinf.2020.05.009.

8. Abrishami A, Samavat S, Behnam B, Arab-Ahmadi M, Nafar M, Sanei TM. Clinical course, imaging features, and outcomes of COVID-19 in kidney transplant recipients. Eur Urol. 2020;6-11. doi: 10.1016/j. eururo.2020.04.064.

9. Akhmerov A, Marban E. COVID-19 and the heart. Circ Res. 2020;126:1443-1455. doi: 10.1161/circresaha.120.317055.
10. Pinto D. Coronavirus disease 2019 (COVID-19): coronary artery disease issues. UpToDate 2019. 2020;1-19.

11. Silva BM, Fonseca AF, Souza SV, Araújo AM, Cardoso MG, Brito $\mathrm{M}$, et al. Effect of high vs low doses of chloroquine diphosphate as adjunctive therapy for patients hospitalized with severe acute respiratory syndrome coronavirus 2 (SARS-CoV-2) infection. JAMA Netw Open. 2020;3:e208857.

12. Mahmud E, Dauerman H, Welt FG, Messenger JC, Rao SV, Grines $\mathrm{C}$, et al. Management of acute myocardial infarction during the COVID-19 pandemic. J Am Coll Cardiol. 2020; doi: 10.1016/j. jacc.2020.04.039.

13. Flexman AM, Abcejo AS, Avitsian R, De Sloovere V, Highton D, Juul N, et al. Neuroanesthesia practice during the COVID-19 pandemic. J Neurosurg Anesthesiol. 2020;32:202-209. doi: 10.1097/ ana.0000000000000691.

14. Li YC, Bai WZ, Hashikawa T. The neuroinvasive potential of SARSCoV2 may play a role in the respiratory failure of COVID-19 patients. J Med Virol. 2020;92:552-555.

15. Bourouiba, L. Turbulent gas clouds and respiratory pathogen emissions: potential implications for reducing transmission of COVID-19. JAMA J Am Med Assoc. 2020; E1-E2. doi: 10.1001/ jama.2020.4756.

16. Zhang Z, Yao W, Wang $\mathrm{Y}$, Long $\mathrm{C}$, Fu X. Wuhan and Hubei COVID-19 mortality analysis reveals the critical role of timely supply of medical resources. J Infect. 2020;81:147-178. doi: 10.1016/j.jinf.2020.03.018. 